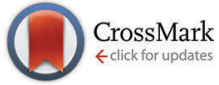

Cite this: Chem. Commun., 2014, 50, 11492

Received 20th June 2014, Accepted 7th August 2014

DOI: $10.1039 / \mathrm{c} 4 \mathrm{cc} 04713 \mathrm{c}$

www.rsc.org/chemcomm

\section{Dual effect of thiol addition on fluorescent polymeric micelles: ON-to-OFF emissive switch and morphology transition $\dagger$}

\author{
Anne B. Mabire, ${ }^{a}$ Mathew P. Robin, ${ }^{a}$ Helen Willcock, ${ }^{a}$ Anaïs Pitto-Barry, ${ }^{a}$ \\ Nigel Kirby ${ }^{b}$ and Rachel K. O'Reilly*a
}

\begin{abstract}
The morphology transition from micelles to vesicles of a solution-state self-assembled block copolymer, containing a fluorescent dye at the core-shell interface, has been induced by an addition-elimination reaction using a thiol, and has been shown to be coupled to a simultaneous ON-to-OFF switch in particle fluorescence.
\end{abstract}

Precise control over solution-state self-assembled polymer morphologies is currently of great interest to the research community. Various morphologies such as spherical micelles, cylinders, rods and vesicles can be formed by the self-assembly of amphiphilic block copolymers in selective solvents. ${ }^{1}$ Block copolymer composition and properties control the morphology that is adopted in solution by the amphiphile. Conventional self-assembled morphologies are based on hydrophilic-hydrophobic repulsive interactions, ${ }^{2}$ and as a result, self-assembled nanostructures formed from stimuli-responsive polymers are able to undergo morphology transitions induced by external stimuli such as $\mathrm{pH}$, temperature and light. ${ }^{3-9}$ Importantly, the responsive behavior of self-assemblies enables these new materials to find applications in nanotechnology and/or drug delivery. ${ }^{10-15}$ Moreover, self-assembled nanostructures having fluorescent properties are also of interest given the desire to track such species in applications such as nanomedicine. ${ }^{16-18}$

Previously, Baker et al. have shown that the conversion of dibromomaleimide (DBM) to dithiomaleimide (DTM) is highly efficient, and that by an excess of thiol further conversions can occur due to retention of the double bond in the DTM motif. ${ }^{19}$ Furthermore, we previously reported that DTM's functionalized with alkyl thiols are highly fluorescent whilst those with aromatic substituents show a significant decrease in fluorescence emission. ${ }^{20}$ Recently, we demonstrated that the DTM motif can be introduced into block copolymers as a highly emissive fluorescent self-reporting

\footnotetext{
${ }^{a}$ Department of Chemistry, University of Warwick, Coventry, CV4 7AL, UK. E-mail: r.k.o-reilly@warwick.ac.uk; Tel: +44 (0)2476523236

${ }^{b}$ Australian Synchrotron, 800 Blackburn Road, Clayton, Victoria 3168, Australia $\dagger$ Electronic supplementary information (ESI) available. See DOI: 10.1039/ c4cc04713c
}

probe via a dual ring-opening polymerization and reversible addition-fragmentation chain transfer polymerization (ROP/ RAFT) initiator. ${ }^{20}$ Aqueous solution-state self-assembly of these amphiphilic block copolymers results in DTM incorporation at the core-shell interface of spherical micelles. ${ }^{21}$ Herein, we utilize the reactivity of the DTM group to induce a morphology transition from spherical micelles to vesicles which occurs simultaneously with an ON-to-OFF switch of fluorescence emission (Fig. 1). The change in fluorescence and morphology is induced by an addition-elimination reaction which removes the hydrophobic segment that is connected to the DTM functional group at the block copolymer interface, and replaces it with an aromatic substituent. This subtle change in chemistry of the DTM group has a significant effect on the properties of the assembly. We propose the use of this triggered change in fluorescence and overall self-assembled structure at the DTM group as an accessible read-out for the change in chemistries within a block copolymer in a polymeric nanostructure. As such we propose this approach has interesting potential scope for use in sensing and also tracking applications.

We first synthesized an amphiphilic block-dye-block copolymer via a combination of $\mathrm{ROP}^{22}$ and RAFT polymerization ${ }^{23}$ utilizing a dual ROP/RAFT initiator, 1 (Scheme 1). The design of this initiator species ensures that the DTM group is located between

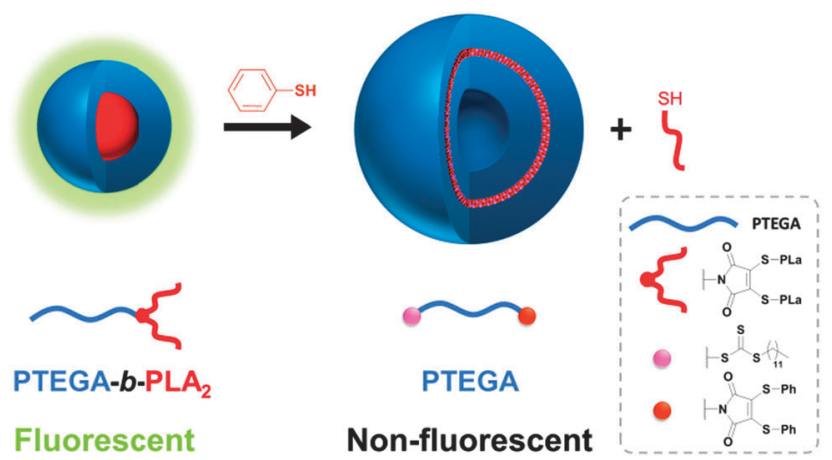

Fig. 1 Schematic of the morphology change induced by an additionelimination reaction of the micelle with thiophenol. 
the hydrophobic and the hydrophilic blocks, allowing the additionelimination reaction to be coupled with both a morphology transition and a fluorescence ON-to-OFF switch. The structure and properties of the amphiphilic copolymers were carefully chosen to enable a significant modification of the hydrophilic/ hydrophobic balance alongside a fluorescence emission decrease by changing the nature of the hydrophobic segment. As the hydrophobic segments were connected to the DTM motif as the thiol ligands, subsequent addition-elimination with a thiol following selfassembly would allow elimination of the hydrophobic blocks.

The DTM-containing ROP/RAFT dual initiator, 1, was synthesized from a 2,3-DBM-functionalized RAFT agent. ${ }^{24}$ Reaction with mercaptoethanol and triethylamine gives a fluorescent DTMfunctionalized ROP/RAFT dual initiator (1) with two hydroxyl groups allowing $\mathrm{ROP}^{22}$ of rac-lactide to be performed to afford polymer $\mathbf{1}^{\prime}$. This polymer was then chain-extended to afford the diblock copolymer [poly(triethyleneglycol monomethyl ether methacrylate) $]-b$-[poly(D,L-lactide) $]_{2}, 2$, see Scheme 1 . The welldefined fluorescent block-dye-block copolymer, PTEGA- $b$-PLA 2,2 , was fully characterized using NMR spectroscopy and SEC analysis $\left(M_{\mathrm{n}}(\mathrm{NMR})=33.1 \mathrm{kDa}, M_{\mathrm{n}}(\mathrm{SEC}, \mathrm{DMF})=19.5 \mathrm{kDa}, \emptyset_{\mathrm{M}}=1.42\right)$.

The self-assembly of copolymer, 2 , into micelles, 3 , was achieved via direct dissolution of the copolymer in purified $18.2 \mathrm{M} \Omega \mathrm{cm}$ water at a concentration of $1 \mathrm{mg} \mathrm{mL}{ }^{-1}$. The fluorescence spectra of the spherical micelles 3 in $18.2 \mathrm{M} \Omega \mathrm{cm}$ water shows an excitation maxima at $405 \mathrm{~nm}$, and an emission maxima at $510 \mathrm{~nm}$, which are similar to previously reported DTM polymer systems. ${ }^{21}$ The size and morphology of the micelles was confirmed by light scattering and microscopy analysis. Multi-angle laser light scattering (MA-LLS) indicated $R_{\mathrm{h}}=26 \mathrm{~nm}$ (see ESI $\dagger$ ) and dry-state transmission electron microscopy (TEM) analysis on graphene oxide, a very thin support that does not require staining, suggests a spherical morphology, see Fig. $2 \mathrm{a}^{25}$

The micelle solution, 3 , was treated with 20 equiv. of thiophenol, and then purified by exhaustive dialysis $(\mathrm{MWCO}=1 \mathrm{kDa})$. Further experiments with a range of aromatic thiols indicated that the
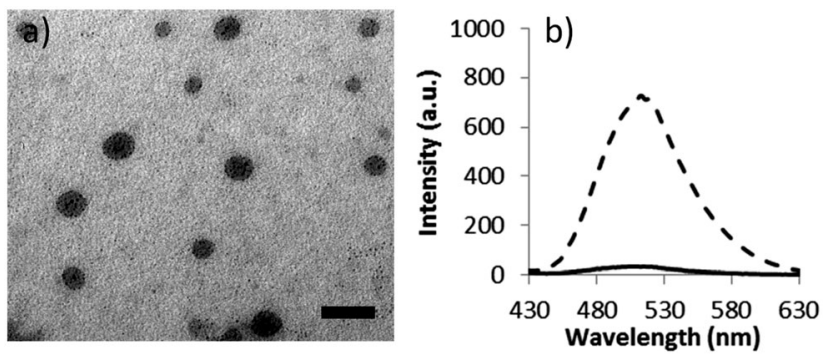

Fig. 2 (a) Representative unstained TEM image of micelles (scale bar = $50 \mathrm{~nm} \mathbf{3}$ and (b) fluorescence emission spectra before (dash line) and after the reaction (solid line), with excitation at $405 \mathrm{~nm}$.

reaction also works efficiently with a small excess of thiol. The thiol underwent addition to the DTM group, with corresponding elimination to afford thio-terminated poly(rac-lactide), 7. Given that the thioPLA residue is insoluble in water, the solution was then centrifuged to remove the thio-PLA precipitate (see ESI $\dagger$ for ${ }^{1} \mathrm{H}$ NMR spectrum).

To characterize the particle morphology of assembly $4 \mathbf{4 a}$, Multi-Angle Laser Light Scattering (MA-LLS) was performed to determine the radius of gyration $R_{\mathrm{g}}$ and hydrodynamic radius $R_{\mathrm{h}}$ of the assemblies, 4a. The ratio of $R_{\mathrm{g}} / R_{\mathrm{h}}$ gives an indication of the nanostructure morphology, with 0.775 indicating a solid micelle and 1 indicating a hollow vesicular structure. ${ }^{26}$ By interpreting the data collected in static light scattering (SLS) mode, using CONTIN analysis, the radius of gyration $R_{\mathrm{g}}$ was determined to be $51 \mathrm{~nm}$. From the dynamic light scattering (DLS) data, $R_{\mathrm{h}}$ was found to be $56 \mathrm{~nm}$. For nanostructure $4 \mathrm{a}$ the $R_{\mathrm{g}} / R_{\mathrm{h}}$ was calculated to be 0.91 , which suggests that nanostructures formed are hollow vesicular particles (see ESI $\dagger$ ). We propose that the vesicles' hydrophobic layer is composed of both the substituted maleimide group (containing the -SPh ligands) and dodecyl end group (the RAFT agent Z-group). This is consistent with previous reports which have shown that hydrophilic polymers with hydrophobic aromatic and aliphatic end-groups can self-assemble into nanoparticles, including vesicles. $^{27-30}$

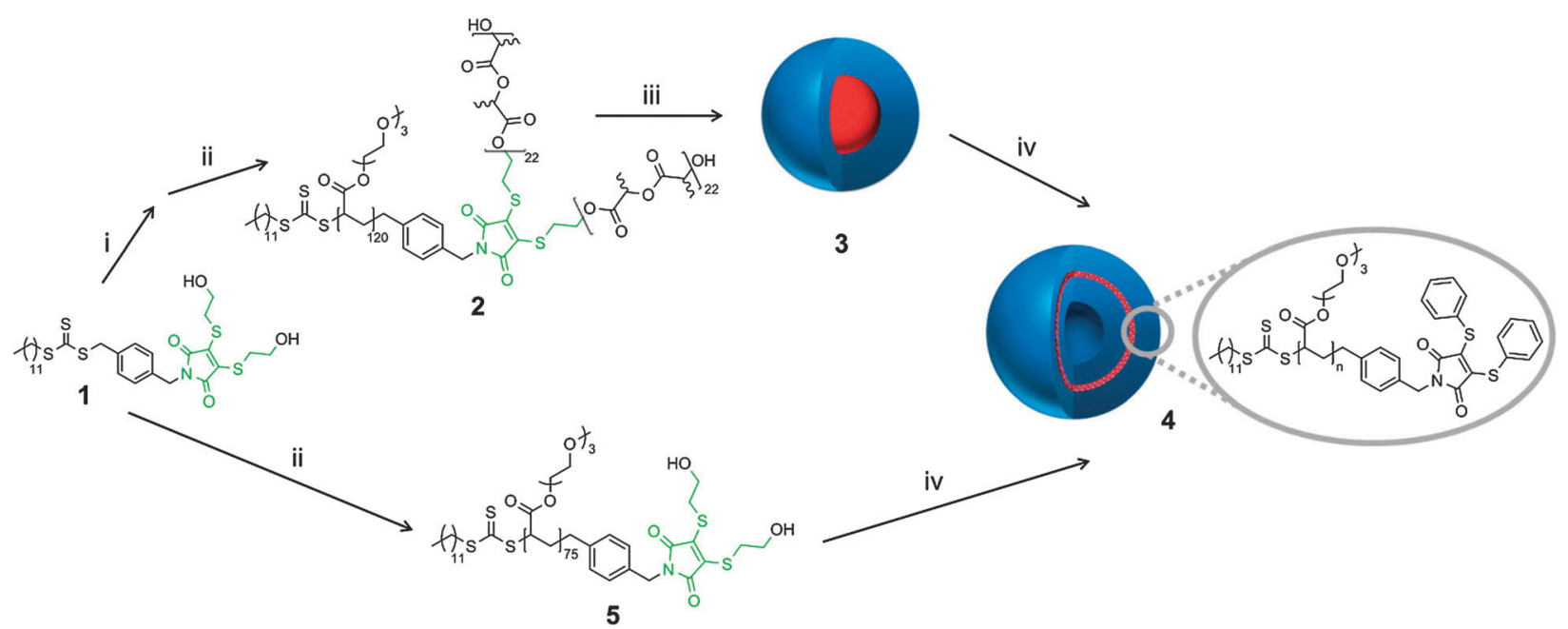

Scheme 1 Preparation of polymers $\mathbf{2}$ and $\mathbf{5}$ from CTA 1, preparation of micelles $\mathbf{3}$, and the addition-elimination reaction of $\mathbf{3}$ and $\mathbf{5}$ which results in a morphology transition to afford vesicle 4 . Conditions: (i) rac-lactide, thiourea, (-)-sparteine, $\mathrm{CH}_{2} \mathrm{Cl}_{2}$; (ii) $\mathrm{AlBN}, \mathrm{CHCl}_{3}, \mathrm{TEGA}$ at $60{ }^{\circ} \mathrm{C}$; (iii) direct dissolution in water; (iv) thiophenol. 


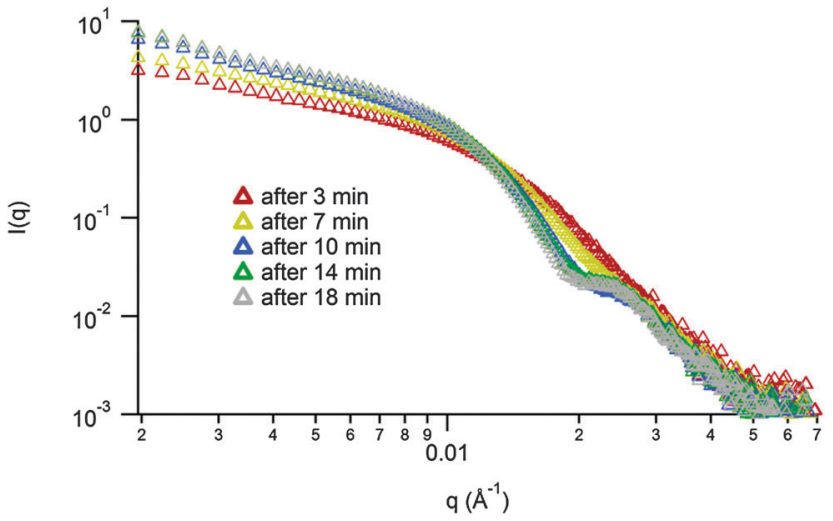

Fig. 3 SAXS profiles for the in situ micelle-to-vesicle transition.

Moreover, examination of the emission spectrum of the resultant solution of $\mathbf{4 a}$ indicated a drastic decrease of the fluorescence as a consequence of the modification at the DTM reactive center. At the same excitation wavelength, the comparison of the emission spectra of the solution before and after the reaction (measured at the same concentration) showed a decrease in the intensity of the maxima $(510 \mathrm{~nm}$ ) from 730 to 30 a.u., see Fig. $2 \mathrm{~b}$.

Unfortunately, attempts to image the vesicles by dry-state TEM were not possible as the nanostructures were not stable to dehydration. However, to further probe the proposed micelleto-vesicle transition upon thiol addition synchrotron SAXS experiments were performed. The transition described in Scheme 1 part (iv) was performed in the beamline and the in situ kinetics of the morphology transition were examined (Fig. 3). This has the advantage that it allows for the monitoring of the transition without the need for removal of insoluble PLA, drying and suspension of the nanostructures (as was necessary for MA-LLS analysis). Analysis of the SAXS curves over a 20 minute time period indicates a change of morphology. At the beginning, a spherical morphology was observed. An increase in the size of the morphologies in solution happened promptly (less than $10 \mathrm{~min}$ ) and a form factor fit indicated the formation of vesicles as well as the presence of random chains in solution (thio-PLA in solution) (see $\mathrm{ESI} \dagger$ for the different fittings of the SAXS curves over time).

To further probe the vesicle formation which was observed in the transition from $\mathbf{3}$ to $\mathbf{4 a}$, the addition-elimination reaction was performed on a range of model homopolymers (data shown for $\mathrm{PTEGA}_{75}, M_{\mathrm{n}}(\mathrm{NMR})=16.2 \mathrm{kDa}, M_{\mathrm{n}}(\mathrm{SEC})=13.5 \mathrm{kDa}, D_{\mathrm{M}}=$ 1.19), 5 , synthesized via RAFT polymerization of TEGA utilizing 1 as a chain transfer agent (CTA). DLS analysis of aqueous solutions of the initial homopolymer 5 (which possesses $\alpha$-diol and $\omega$-dodecyl end-groups), indicated the presence of unimers in solution. However, after the addition-elimination reaction between $\mathbf{5}$ and an excess of thiophenol, well-defined nanostructures were observed, $4 \mathbf{b}$. MA-LLS was performed, and values of $R_{\mathrm{g}}$ and $R_{\mathrm{h}}$ were extracted from the results. By interpreting the data collected in SLS mode, the $R_{\mathrm{g}}$ equals $63 \mathrm{~nm}$ and from the DLS data, the $R_{\mathrm{h}}$ equals $68 \mathrm{~nm}$ and hence the $R_{\mathrm{g}} / R_{\mathrm{h}}$ obtained was 0.93 , which once again suggests that the nanostructures formed in this reaction are vesicles. Similar to particles 4a which result from reaction of the micelles (3) with thiophenol, the reaction of the homopolymer 5 with thiophenol forms particles $\mathbf{4 b}$ of a similar size and morphology. This is understandable as the addition-elimination reaction of $\mathbf{3}$ and 5 would be expected to give the same resultant homopolymer, namely P(TEGA) homopolymer with $\alpha$-SPh and $\omega$-dodecyl endgroups, which would be expected to assemble into a similar morphology. As observed for the addition-elimination reaction with the micelles, the fluorescence emission of the homopolymer solution again underwent a fast (15 min by analysis of the $535 \mathrm{~nm}$ emission) ON-to-OFF switch during the reaction with thiophenol, see ESI. $\dagger$

In conclusion, we have shown that an amphiphilic blockdye-block copolymer containing a DTM group can undergo a fast morphology transition from spherical micelles to vesicles. This is triggered by the addition of thiophenol to the DTM group, with corresponding elimination of the hydrophobic blocks. A unimer-to-vesicle transition also occurs for a DTM groupcontaining homopolymer. In both cases addition of thiophenol leads to a simultaneous fluorescence ON-to-OFF switch. This approach is extremely versatile and could be tuned for utilization with a range of aromatic thiols and self-assembled systems which contain the DTM functional group. We suggest that such a simultaneous $\mathrm{ON}-\mathrm{OFF}$ switch and morphological reorganization could be readily applied as a tracking ingmechanism and also as a mechanism for monitoring release in biological and/or synthetic self-assembled systems.

We are thankful to the EPSRC and the IAS at the University of Warwick for funding. Equipment used in this research was funded in part through Advantage West Midlands (AWM) Science City Initiative and in part by the ERDF. We acknowledge Professor Andrew Dove and Dr Tara Schiller for assistance with the SAXS measurements and Mr Daniel Wright for assistance with MA-LLS analysis.

\section{Notes and references}

1 Y. Mai and A. Eisenberg, Chem. Soc. Rev., 2012, 41, 5969-5985.

2 A. Blanazs, S. P. Armes and A. J. Ryan, Macromol. Rapid Commun., 2009, 30, 267-277.

3 A. O. Moughton and R. K. O'Reilly, Chem. Commun., 2010, 46, 1091-1093.

4 J.-Z. Du, H.-Y. Long, Y.-Y. Yuan, M.-M. Song, L. Chen, H. Bi and J. Wang, Chem. Commun., 2012, 48, 1257-1259.

5 A. Klaikherd, C. Nagamani and S. Thayumanavan, J. Am. Chem. Soc., 2009, 131, 4830-4838.

6 C. L. McCormick, B. S. Sumerlin, B. S. Lokitz and J. E. Stempka, Soft Matter, 2008, 4, 1760-1773.

7 F. Chécot, S. Lecommandoux, Y. Gnanou and H.-A. Klok, Angew. Chem., Int. Ed., 2002, 41, 1339-1343.

8 C. Chang, H. Wei, J. Feng, Z.-C. Wang, X.-J. Wu, D.-Q. Wu, S.-X. Cheng, X.-Z. Zhang and R.-X. Zhuo, Macromolecules, 2009, 42, 4838-4844.

9 W. Kim, J. Thévenot, E. Ibarboure, S. Lecommandoux and E. L. Chaikof, Angew. Chem., Int. Ed., 2010, 49, 4257-4260.

10 M. Lazzari and M. A. López-Quintela, Adv. Mater., 2003, 15, 1583-1594.

11 G. Gaucher, M.-H. Dufresne, V. P. Sant, N. Kang, D. Maysinger and J.-C. Leroux, J. Controlled Release, 2005, 109, 169-188.

12 Z. L. Tyrrell, Y. Shen and M. Radosz, Prog. Polym. Sci., 2010, 35, 1128-1143.

13 K. Miyata, R. J. Christie and K. Kataoka, React. Funct. Polym., 2011, 71, 227-234.

14 E. G. Kelley, J. N. L. Albert, M. O. Sullivan and T. H. Epps, III, Chem. Soc. Rev., 2013, 42, 7057-7071. 
15 M. Elsabahy and K. L. Wooley, Chem. Soc. Rev., 2012, 41, 2545-2561. 16 M. J. Ruedas-Rama, J. D. Walters, A. Orte and E. A. H. Hall, Anal. Chim. Acta, 2012, 751, 1-23.

17 F. Canfarotta, M. J. Whitcombe and S. A. Piletsky, Biotechnol. Adv., 2013, 31, 1585-1599.

18 S. M. Janib, A. S. Moses and J. A. MacKay, Adv. Drug Delivery Rev., 2010, 62, 1052-1063.

19 M. E. B. Smith, F. F. Schumacher, C. P. Ryan, L. M. Tedaldi, D. Papaioannou, G. Waksman, S. Caddick and J. R. Baker, J. Am. Chem. Soc., 2010, 132, 1960-1965.

20 M. P. Robin, P. Wilson, A. B. Mabire, J. K. Kiviaho, J. E. Raymond, D. M. Haddleton and R. K. O'Reilly, J. Am. Chem. Soc., 2013, 135, 2875-2878.

21 M. P. Robin, A. B. Mabire, J. C. Damborsky, E. S. Thom, U. H. Winzer-Serhan, J. E. Raymond and R. K. O'Reilly, J. Am. Chem. Soc., 2013, 135, 9518-9524.

22 R. C. Pratt, B. G. G. Lohmeijer, D. A. Long, P. N. P. Lundberg, A. P. Dove, H. Li, C. G. Wade, R. M. Waymouth and J. L. Hedrick, Macromolecules, 2006, 39, 7863-7871.
23 G. Moad, E. Rizzardo and S. H. Thang, Aust. J. Chem., 2009, 62, 1402-1472.

24 M. P. Robin, M. W. Jones, D. M. Haddleton and R. K. O'Reilly, ACS Macro Lett., 2012, 1, 222-226.

25 J. P. Patterson, A. M. Sanchez, N. Petzetakis, T. P. Smart, T. H. Epps, III, I. Portman, N. R. Wilson and R. K. O'Reilly, Soft Matter, 2012, 8, 3322-3328.

26 J. P. Patterson, M. P. Robin, C. Chassenieux, O. Colombani and R. K. O'Reilly, Chem. Soc. Rev., 2014, 43, 2412-2425.

27 J. Du, H. Willcock, J. P. Patterson, I. Portman and R. K. O'Reilly, Small, 2011, 7, 2070-2080.

28 J. Xu, L. Tao, C. Boyer, A. B. Lowe and T. P. Davis, Macromolecules, 2010, 44, 299-312.

29 J. P. Patterson, E. G. Kelley, R. P. Murphy, A. O. Moughton, M. P. Robin, A. Lu, O. Colombani, C. Chassenieux, D. Cheung, M. O. Sullivan, T. H. Epps, III and R. K. O'Reilly, Macromolecules, 2013, 46, 6319-6325.

30 T. Liu, W. Tian, Y. Zhu, Y. Bai, H. Yan and J. Du, Polym. Chem., 2014, 5, 5077-5088. 